\title{
Computation of the Autocovariances for Time Series with Multiple Long-Range Persistencies
}

\author{
Tucker S. McElroy* and Scott H. Holan ${ }^{\dagger \ddagger}$
}

\begin{abstract}
Gegenbauer processes allow for flexible and convenient modeling of time series data with multiple spectral peaks, where the qualitative description of these peaks is via the concept of cyclical longrange dependence. The Gegenbauer class is extensive, including ARFIMA, seasonal ARFIMA, and GARMA processes as special cases. Model estimation is challenging for Gegenbauer processes when multiple zeroes and poles occur in the spectral density, because the autocovariance function is laborious to compute. The method of splitting - essentially computing autocovariances by convolving long memory and short memory dynamics - is only tractable when a single long memory pole exists. The main contribution of this paper is to propose an additive decomposition of the spectrum into a sum of spectra that each have a single singularity, so that a computationally efficient splitting method can be applied to each term and then aggregated. This approach differs from McElroy and Holan (2012), which handles all poles in the spectral density at once through a careful analysis of truncation error. This paper's technique allows for fast estimation of time series with multiple long-range dependencies, which is illustrated numerically and through several case-studies.
\end{abstract}

Keywords: Gegenbauer; Long memory; Long-range dependence; Quasi-biennial oscillations; Seasonal long memory; Spectral density.

\footnotetext{
${ }^{*}$ Center for Statistical Research and Methodology, U.S. Census Bureau, 4600 Silver Hill Road, Washington, D.C. 20233-9100, tucker.s.mcelroy@census.gov

${ }^{\dagger}$ Department of Statistics, University of Missouri, 146 Middlebush Hall, Columbia, MO, 65211-6100, holans@missouri.edu

${ }^{\ddagger}$ Disclaimer: This paper is released to inform interested parties of ongoing research and to encourage discussion of work in progress. The views expressed are those of the authors and not necessarily those of the U.S. Census Bureau.
} 


\section{Introduction}

This paper is concerned with the modeling of time series data with multiple persistent periodicities, where the persistency indicates a slow rate of decay in sample autocorrelations. Many time series of interest in macroeconomics, finance, and meteorology have persistency at nonzero frequencies, which are properly described as infinite (but integrable) peaks in the spectral density; see Ferrara and Guégan (2000), Bisaglia et al. (2003), Soares and Souza (2006), Talamantes et al. (2007), and Gil-Alana (2008). When two or more such peaks are present in the spectrum of the underlying process, the commonly used sets of models - ARFIMA (Hosking, 1981), seasonal ARFIMA (PorterHudak, 1990), Gegenbauer (Gray et al. 1989), etc. - are challenging to compute. That is, the autocovariance function of such models is laborious to calculate, although in the case of a single long memory spectral peak there exists certain strategies that facilitate computation. Recently, McElroy and Holan (2012) developed a general representation for processes with multiple spectral peaks, and showed how one can accurately determine the Wold coefficients of such processes from the cepstral representation (Bloomfield, 1973). Further, McElroy and Holan (2012) produced error terms (expressed as hypergeometric functions) that accounted for truncation error in the calculation of autocovariances. The current paper presents a different algorithm that is both fast and accurate, and goes back to the older "splitting" method of Bertelli and Caporin (2002).

Briefly, in a nonparametric or semiparametric conception of the spectral density $f$, in which possibly several persistent phenomena exist, it is natural to write $f$ as a product of functions that represent the poles in the spectrum, together with a term that corresponds to short memory dynamics, being both bounded and bounded away from zero. In our discussion, we may also be interested in anti-persistent behavior, or negative memory (McElroy and Politis, 2014), which corresponds to spectral troughs; i.e., a zero in $f$. The autocovariance sequence corresponding to $f$

is equal to the convolution of the autocovariances of each term in the product; if there is only one pole/zero in the spectral density, then we must convolve the corresponding autocovariance function with that of the short memory portion, which can be safely truncated. This truncation of the short memory autocovariance function - presuming that the long memory autocovariance function is known analytically, or can be calculated to high accuracy - is called the splitting method, and is developed in Bertelli and Caporin (2002) and Hurvich (2002).

This ingenious algorithm does not extend directly to having two or more poles/zeroes, because then there are at least three functions being convolved, which means there are at least two infinite 
summations - and there seems to be no way to truncate these without a potentially tremendous loss of accuracy. The main insight of this paper is that a function $f$ with $k \geq 1$ poles/zeroes can be re-expressed as the sum of $k$ distinct functions, each of which has a single pole/zero, together with its own distinct short memory spectrum. This is akin to a partial fraction decomposition, though instead of polynomials, we must work with somewhat more complicated frequency domain functions. The splitting method can be applied to each of the $k$ distinct terms, and the corresponding autocovariances simply summed up.

This algorithm allows us to consider a potentially large number of poles/zeroes. In fact, in one of our illustrations (Section 5), we have $k=26$ seasonal poles! This is done without much worry about the potential loss of accuracy due to truncation, because we only have the approximation error due to the splitting method, which is typically negligible due to the rapid decay of autocovariances of short memory processes. Thus, complex time series data with multiple persistencies (where the frequency of persistency may be either unknown - in which case it is a parameter to be estimated - or known) can now be readily treated, whereas previously only special cases (e.g., seasonal ARFIMA, or the two-pole cases of McElroy and Holan (2012)) could realistically be handled. We demonstrate this algorithmic breakthrough on a broad variety of series.

The resulting algorithms have been implemented for both a Gaussian likelihood and a Whittle likelihood, where the short memory portion is semiparametrically described via the device of a cepstral model - this is just a Fourier expansion of the log spectrum. The aforementioned algorithms have been coded using the R programming language (R Core Team, 2014) and are available upon request from the first author. Adapting them to versions where the short memory portion is described via an ARMA process is straightforward, and thus is not pursued here 1 . Another application would be to consider several latent components, each of which are potentially cyclically persistent. In this case, the autocovariance of the observed process is given as the sum of the autocovariances associated with each latent process, as described in a more limited context in Holan and McElroy (2012).

The focus of this paper is primarily algorithmic/computational and can be adapted across a wide-array of statistical models. Specifically, the algorithms proposed herein should be useful to any of the cyclical long memory models currently being utilized to analyze time series with multiple persistencies. Section 2 provides the general semiparametric framework and shows how

\footnotetext{
${ }^{1}$ Note that equation (4.3) of McElroy and Holan (2012), which relates the cepstral coefficients to the ARMA roots, has a sign error.
} 
popular models can be embedded therein. Section 3 thoroughly develops the algorithm, whereas Section 4 presents numerical studies of speed and accuracy. We provide several illustrations in Section 5, utilizing our algorithm to fit data with multiple persistencies across a variety of scientific disciplines. Concluding discussion is provided in Section 6, with derivations left to an Appendix.

\section{A Semiparametric Description of Multiple Persistencies}

In this paper we focus on the $k$-GEXP process, which amounts to incorporating zeroes and poles in the spectral density, together with a cepstral description of the short memory. Ths short memory dynamics could also be described by an ARMA process, resulting in a multi-pole extension of the ARFIMA; if readers are interested in this variant, the results of Section 3 (namely, the computation of autocovariances corresponding to the spectrum $g$ ) could be modified accordingly.

The $k$-GEXP model was introduced in McElroy and Holan (2012), and is recapitulated here. Let $\left\{X_{t}\right\}$ be a mean zero covariance stationary time series. We will suppose that $\left\{X_{t}\right\}$ follows a $k$-factor Generalized Exponential model, or $k$-GEXP model, which is for convenience defined in the frequency domain 2 . (The $k$-GEXP model is a generalization of the simpler EXP model, allowing for poles in the spectrum at any frequency between 0 and $\pi$.) The $k$-GEXP spectral density can be written compactly as

$$
f(\lambda)=f_{0}^{-a}(\lambda) f_{\pi}^{-b}(\lambda) \prod_{j=1}^{k} f_{\omega_{j}}^{-c_{j}}(\lambda) g(\lambda),
$$

where the functions of frequency $f_{\vartheta}$ are defined via

$$
f_{\vartheta}(\lambda)=\left\{\begin{array}{l}
\left|1-e^{-i \lambda}\right|^{2} \quad \text { if } \vartheta=0 \\
\left|1+e^{-i \lambda}\right|^{2} \quad \text { if } \vartheta=\pi \\
\left|1-e^{-i \vartheta} e^{-i \lambda}\right|^{2}\left|1-e^{i \vartheta} e^{-i \lambda}\right|^{2} \quad \text { if } \vartheta \neq 0, \pi
\end{array} .\right.
$$

and the function $g$ is bounded, representing the short memory portion of the spectrum. Also, the parameters $a, b, c_{1}, \ldots, c_{k}$ are each bounded in $(-1 / 2,1 / 2)$ in order to guarantee stationarity. The frequencies $\omega_{\ell}$ are distinct from one another, and not equal to zero or $\pi$. When a parameter $a, b$, or $c_{j}$ is positive, there is a corresponding pole in the spectral density at frequency zero, $\pi$, or $\omega_{j}$

\footnotetext{
${ }^{2}$ The infinite moving average representation of a $k$-GEXP is discussed in McElroy and Holan (2012), with coefficients $\left\{\psi_{j}\right\}$ such that $X_{t}=\sum_{j \geq 0} \psi_{j} \epsilon_{t-j}$ for some white noise (possible non-Gaussian) process $\left\{\epsilon_{t}\right\}$. The coefficients $\left\{\psi_{j}\right\}$ of this time domain representation of the $k$-GEXP cannot be easily expressed - as in the ARFIMA case - but can be computed from the spectral density.
} 
respectively - this is the case of long memory. On the other hand, negative parameters correspond to a zero in the spectrum, and correspond to intermediate memory (or negative memory, also called anti-persistence by some authors; see Beran (2010) and the references therein). Note that the total number of poles/zeroes in (1) is at most $k+2$, with a factor being omitted when the corresponding persistency parameter is zero.

The short memory spectrum $g$ corresponds to an $\operatorname{EXP}(q)$ model (Bloomfield, 1973) so that

$$
g(\lambda)=\sigma^{2} \exp \left\{\sum_{j=1}^{q} g_{j} \cos (\lambda j)\right\}=\exp \left\{g_{0}+\frac{1}{2} \sum_{0<|j| \leq q} g_{j} e^{-i \lambda j}\right\}
$$

where $g_{-j}=g_{j}$. So the innovation variance $\sigma^{2}$ of the model is equal to $\exp \left(g_{0}\right)$. Here $q$ can be viewed as a finite integer, with the case $q=\infty$ allowing for an arbitrary invertible short memory spectral density. Putting together the short memory and long memory portions yields the $k$ $\operatorname{GEXP}(q)$ model: $k+2$ parameters govern the degree of persistence, with up to another $k+2$ parameters governing the peak locations (when they are not known a priori) or periodicities, and $q$ parameters governing the short memory dynamics. Typically a mean parameter would also be utilized, whereas the innovation variance can be estimated utilizing a profile likelihood. Note that, even though the model is formulated in the frequency domain, estimation of parameters can be done in the time domain (by Gaussian likelihood maximization) or in the frequency domain (by Whittle likelihood minimization).

By shifting the various $\omega_{\ell}$, one can capture a variety of quasi-cyclical phenomena, such as seasonality, cyclicality, and long-term movements in the process. For a mean-zero Gaussian process, the spectrum $f$ contains all of the model parameters of interest, so that one only needs to compute the autocovariances, i.e., the inverse Fourier Transform (FT), of the spectrum in order to determine the Gaussian likelihood function. These autocovariances are necessary for both frequentist and Bayesian approaches to model estimation, and are also required for forecasting applications. Therefore, it is vital to have an efficient method of computing autocovariances for any given values of the associated model parameters.

One problem with the model as it stands, is that it is not identifiable when $k>1$. This is because we can swap any two frequencies $\omega_{1}, \omega_{2}$, along with their corresponding memory parameters, and obtain the exact same value of the spectral density. In order to guarantee identifiability in practice, one might put a priori restrictions on the frequencies $\omega_{\ell}$, such as insisting that each one belongs to a disjoint subset of $(0, \pi)$. In our own implementations, we ensure that the various $\omega_{k}$ are distinct 
via the following parametrization: for $\theta_{1}, \theta_{2}, \ldots, \theta_{k} \in \mathbb{R}$, and $\theta_{0}=0$, set

$$
\omega_{j+1}=\frac{\pi \exp \left(\theta_{j+1}\right)+\omega_{j}}{\exp \left(\theta_{j+1}\right)+1}
$$

for $j=0,1, \ldots, k-1$. This ensures that $\omega_{j+1} \in\left(\omega_{j}, \pi\right)$.

For many applications of the model, the frequencies are known ahead of time and therefore are not even parameters. For example, extremely persistent cyclical effects typically correspond to trends or seasonality, for which the corresponding frequencies are known. Seasonality is a term referring to a situation where the sampling frequency relative to annual time units is an integer. If there are $p$ seasons, where $p$ is integer, and the sampling frequency coincides with $p$, then seasonality might manifest in the data. If seasonality is present, then it can often be described through peaks in the spectral density at frequencies $2 \pi j / p$ for $1 \leq j \leq p$. Trend, on the other hand, is often described through polynomial functions or stochastic components, and tends to contribute spectral mass at the low frequencies.

However, there may be some occasions where the cyclical frequencies are not known ahead of time. For phenomena that are not tied to the terrestrial cycle (approximately 365 days), the sampling frequency may have no special meaning in terms of seasons. For astronomical data, the existence of long-range cycles with strong persistence is a known phenomenon, where the period can involve non-integer multiples of years. One of our case studies involves a time series of wind measurements, with dynamics that are well-captured using a model specification that exhibits longrange persistencies - one of which does not correspond to a regular seasonal effect. Such phenomena might manifest as peaks in the spectral density, but the peak frequency is not known a priori, and must be estimated.

A secondary issue is weak identifiability problems due to possible confounding of the short memory $g$ and the zero-pole (ZP) contributions to (1). Because values of the exponents close to zero make the spectrum approximately flat, excepting a kink, the same behavior can be approximated by the cepstral coefficients. In finite samples it is possible to have confusion between these elements, and therefore we have found it advisable to keep the cepstral order low (i.e., $q \leq 5$ ). In practice, this issue is not terribly important, because highly persistent peaks/troughs have a distinct spectral shape that is difficult to capture, or even approximate, with a short memory spectral density. 


\section{Derivation of the New Algorithm}

A key challenge in the computation of the autocovariance function (acf) for multiple-pole long memory processes is the trade-off between computational efficiency and accuracy mentioned in McElroy and Holan (2012); truncation techniques attempt to balance the horns of this dilemma. A splitting method, such as that proposed in Bertelli and Caporin (2002) and Hurvich (2002), can be successful when there is only a single long memory pole in the spectrum; but with multiple poles in the spectrum, long memory autocovariances become convolved with other long memory autcovariances, implying that truncation of the convolution involves a substantial approximation error. The novel idea of this paper is to factor the multi-pole form of the spectrum into a sum of several components, each of which only contains a single long memory pole. Then each component can have its autocovariances calculated via splitting, and the results summed to produce the desired outcome.

Some notation that we require is given next. We use $z=e^{-i \lambda}$ where $\lambda \in[-\pi, \pi]$, and for any real-valued function $g(\lambda)$, its inverse FT is defined to be the (possibly complex) sequence $\gamma_{h}(g)$, defined via the formula

$$
\gamma_{h}(g)=\frac{1}{2 \pi} \int_{-\pi}^{\pi} g(\lambda) z^{-h} d \lambda .
$$

Conversely, the FT of a sequence $\left\{a_{h}\right\}$ is the function $\sum_{h=-\infty}^{\infty} a_{h} z^{h}$. This establishes our conventions concerning $2 \pi$. In the case that $g$ is even, its inverse FT sequence is symmetric. Furthermore, if $g$ is the spectral density of a time series, its inverse FT is interpretable as the acf of the process.

The key result is a factorization that we describe below, which is akin to a partial fraction decomposition of the spectrum. Let $d^{+}=\max \{d, 0\}$ and $d^{-}=\min \{d, 0\}$ where $d$ can be any of the exponents $a, b, c_{j}$ - if a particular term is not present in the spectrum, this is handled by setting the exponent to zero. So $d^{+}+d^{-}=d$ throughout. Then, apart from the short memory portion $g$, we can split $f$ into two factors $f^{-}$and $f^{+}$corresponding to the terms having zeroes and the terms having poles, respectively. That is, $f=f^{-} f^{+} g$ with

$$
\begin{aligned}
& f^{-}=f_{0}^{-a^{-}} f_{\pi}^{-b^{-}} \prod_{j=1}^{k} f_{\omega_{j}}^{-c_{j}^{-}} \\
& f^{+}=f_{0}^{-a^{+}} f_{\pi}^{-b^{+}} \prod_{j=1}^{k} f_{\omega_{j}}^{-c_{j}^{+}} .
\end{aligned}
$$

We wish to factorize $f$ into a sum of ZP terms that each involve a single component of the $f_{\omega}$ terms, together with a short memory portion. Toward that end, we define the ZP "version" of the 
spectrum via

$$
f_{Z P}=f_{0}^{-a} 1_{\{a \neq 0\}}+f_{\pi}^{-b} 1_{\{b \neq 0\}}+\sum_{j=1}^{k} f_{\omega_{j}}^{-c_{j}} 1_{\{k>0\}} .
$$

With this notation, we can state the algebraic factorization result.

Proposition 1 Given a k-GEXP spectrum of the form (1) and factors given by (2), there exists a short memory spectrum $\nu$ such that

$$
\begin{aligned}
\nu & =\left(f_{0}^{-a^{-}} f_{\pi}^{b^{+}} 1_{\{a \neq 0\}}+f_{\pi}^{-b^{-}} f_{0}^{a^{+}} 1_{\{b \neq 0\}}\right) \prod_{j=1}^{k} f_{\omega_{j}}^{c_{j}^{+}}+f_{0}^{a^{+}} f_{\pi}^{b^{+}} \sum_{j=1}^{k} \prod_{\ell \neq j} f_{\omega_{\ell}}^{c_{\ell}^{+}} f_{\omega_{j}}^{-c_{j}^{-}} 1_{\{k>0\}}, \\
\tau & =f^{-} \cdot g / \nu \\
f & =\tau \cdot f_{Z P} .
\end{aligned}
$$

The function $\nu$ is bounded, and is also strictly positive if there are at least two ZP factors - in which case it corresponds to a short memory process. The function $\tau$ has only short memory and negative memory (i.e., it has no poles).

This factorization yields a decomposition of $f$ into a sum of up to $k+2$ terms, each of which involves $\tau$ divided by a factor $f_{\vartheta}^{-d}$. Therefore, the inverse FT of each summand of $f$ can be computed by convolving the inverse FT of the numerator $\tau$ and denominator $f_{\vartheta}^{-d}$ via the splitting method. This is advantageous, because: (a) exact formulas for the acfs of the ZP factors $f_{\omega}^{-d}$ are known, and (b) the inverse FT for $\tau$ can be calculated to decent approximation (using techniques of McElroy and Holan (2012)) since they have short/negative memory. The formulas needed are summarized in the following result, wherein we let $\Gamma$ and $F$ denote the Gamma function and hypergeometric function, respectively.

Proposition 2 The autocovariance sequence of the ZP factor $\tau / f_{\vartheta}^{d}$ is given by the following formulas:

$$
\begin{aligned}
\gamma_{h}\left(\tau / f_{\vartheta}^{d}\right)= & \sum_{j=-\infty}^{\infty} \gamma_{j}(\tau) \gamma_{h+j}\left(f_{\vartheta}^{-d}\right), \\
\gamma_{h}\left(f_{\vartheta}^{-d}\right)= & \left\{\begin{array}{l}
\frac{\Gamma(h+d) \Gamma(1-2 d)}{\Gamma(h-d+1) \Gamma(1-d) \Gamma(d)}, \quad \text { if } \vartheta=0 \\
(-1)^{h} \frac{\Gamma(h+d) \Gamma(1-2 d)}{\Gamma(h-d+1) \Gamma(1-d) \Gamma(d)}, \quad \text { if } \vartheta=\pi \\
\sqrt{\pi} \Gamma(1-2 d)(2 \sin \vartheta)^{1 / 2-2 d}\left(P_{h-1 / 2}^{2 d-1 / 2}(\cos \vartheta)+(-1)^{h} P_{h-1 / 2}^{2 d-1 / 2}(-\cos \vartheta)\right), \quad \text { if } \vartheta \neq 0, \pi
\end{array}\right. \\
P_{h-1 / 2}^{2 d-1 / 2}(z)= & \left(\frac{2 h-2}{h-2 d}\right) z P_{h-3 / 2}^{2 d-1 / 2}(z)-\left(\frac{2 d+h-2}{h-2 d}\right) P_{h-5 / 2}^{2 d-1 / 2}(z) .
\end{aligned}
$$


The functions $P^{2 d-1 / 2}(z)$ are Legendre functions, and their recursive definition is initialized by

$$
\begin{aligned}
& P_{-1 / 2}^{2 d-1 / 2}(z)=\left(\frac{1+z}{1-z}\right)^{d-1 / 4} \frac{F(1 / 2,1 / 2,3 / 2-2 d ;(1-z) / 2)}{\Gamma(3 / 2-2 d)} \\
& P_{1 / 2}^{2 d-1 / 2}(z)=\left(\frac{1+z}{1-z}\right)^{d-1 / 4} \frac{F(-1 / 2,3 / 2,3 / 2-2 d ;(1-z) / 2)}{\Gamma(3 / 2-2 d)} .
\end{aligned}
$$

The first formula of Proposition 2, namely the convolution of the short memory $\tau$ autocovariances with the long/negative memory ZP autocovariances, is called a "splitting formula." We can truncate the splitting formula at some threshold lag $J$ without much cost to accuracy, because the $\left\{\gamma_{j}(\tau)\right\}$ sequence is absolutely summable, and hence decays rapidly. However, one caution is that the truncation of $\left\{\gamma_{j}(\tau)\right\}$ at $J$ (i.e., setting $\gamma_{j}(\tau)=0$ for $j>J$ ) can result in a sequence that is not positive definite, such that the approximation to the inverse FT

$$
\gamma_{h}\left(\tau / f_{\vartheta}^{d}\right) \approx \gamma_{0}(\tau) \gamma_{h}\left(f_{\vartheta}^{-d}\right)+\sum_{j=1}^{J} \gamma_{j}(\tau)\left\{\gamma_{h+j}\left(f_{\vartheta}^{-d}\right)+\gamma_{h-j}\left(f_{\vartheta}^{-d}\right)\right\}
$$

need not be positive definite. This may seem to be a quibble, but we have found it to be quite important in practice, because a nonpositive definite sequence implies negative eigenvalues in the corresponding Toeplitz covariance matrix, obviating efforts at inversion. Our remedy is to construct a $J$-dependent process that has well-defined spectral density that closely approximates $\tau$. One way to do this is to determine the Wold coefficients of $\tau$ (obtained via determining the cepstral coefficients, and utilizing the recursions described in Hurvich (2002)), and define $\tau^{(J)}$ to be spectral density of the $\operatorname{MA}(J)$ process corresponding to retaining only the first $J+1$ Wold coefficients (the first Wold coefficient is always equal to one). Letting $\xi_{k}=2 \pi^{-1} \int_{0}^{\pi} \log \tau(\lambda) \cos (\lambda k) d \lambda$ be the $k$ th cepstral coefficient, for $k \geq 1$, we have Pourahmadi's recursions (Pourahmadi, 1984) involving the Wold coefficients $\psi_{j}\left(\right.$ with $\left.\psi_{0}=1\right)$ :

$$
\psi_{j}=\frac{1}{2 j} \sum_{k=1}^{j} \psi_{j-k} k \xi_{k}
$$

Then $\tau^{(J)}(\lambda)=\left|\sum_{k=0}^{J} \psi_{k} e^{-i \lambda k}\right|^{2}$ by definition, and its autocovariance sequence is guaranteed to be positive definite; hence we utilize the approximation

$$
\gamma_{h}\left(\tau / f_{\vartheta}^{d}\right) \approx \gamma_{0}\left(\tau^{(J)}\right) \gamma_{h}\left(f_{\vartheta}^{-d}\right)+\sum_{j=1}^{J} \gamma_{j}\left(\tau^{(J)}\right)\left\{\gamma_{h+j}\left(f_{\vartheta}^{-d}\right)+\gamma_{h-j}\left(f_{\vartheta}^{-d}\right)\right\} .
$$

There is no need to take $J$ particularly large in practice, due to the rapid decay rate of the Wold coefficients of $\tau(z)$ - typically $J=10$ is more than sufficient. We note that this approach to 
obtaining the $J$-dependent approximation is practicable, because the $j$ th Wold coefficient only depends (recursively) upon the first $j$ cepstral coefficients, as shown in Pourahmadi (1984) and Hurvich (2002). Therefore, one only needs the first $J$ cepstral coefficients (we compute these using numerical integration, which is extremely accurate for short memory spectra). Using the recursions to obtain the first $J$ Wold coefficients, one then computes the autocovariances of this $\mathrm{MA}(J)$ process.

Explicit steps of the entire algorithm are given as follows. Choose a truncation level $J$, and a total number of lags $H$ for the acf sequence.

1. Read in the parameters.

2. Compute $g(\lambda), f^{-}(\lambda), \nu(\lambda)$, and $\tau(\lambda)$ for a mesh of $\lambda$ frequencies.

3. The $j$ th cepstral coefficient of $\tau$ is $2 \int_{0}^{1} \log \tau(\pi x) \cos (\pi j x) d x$, which can be Riemann approximated at a given mesh.

4. Obtain the Wold coefficients $\psi_{j}$ for $1 \leq j \leq J$ of $\tau$ from the cepstral coefficients via (5), and compute the autocovariances of $\tau^{(J)}$ from these Wold coefficients.

5. The acf of each long/negative memory pole is given by the direct formula in Proposition 2 .

6. Combine the acfs by convolution (6).

This algorithm might be used to do forecasting, taking the parameters in the first step to be known estimates; or this algorithm could be embedded in a routine to compute the Gaussian likelihood, in which case the parameters in Step 1 reflect the current iterates in a numerical optimization scheme. In the case of the Gaussian likelihood, the innovations algorithm (Brockwell and Davis, 1991) can be utilized, which has a loop over the sample size and is recursive in nature; similarly, a variant for the Whittle likelihood is available. The mean-zero scaled log Gaussian likelihood, based on a sample of size $n$ from the data process - denoted by $X=\left[X_{1}, X_{2}, \cdots, X_{n}\right]^{\prime}-$ is

$$
\mathcal{L}(\varphi ; X)=X^{\prime} \Sigma^{-1}\left(f_{\varphi}\right) X+\log \operatorname{det} \Sigma\left(f_{\varphi}\right)
$$

where $\Sigma\left(f_{\varphi}\right)$ denotes the $n \times n$ Toeplitz covariance matrix corresponding to the spectral density $f_{\varphi}$, and $\varphi$ abbreviates the full list of model parameters excluding the mean (in our applications, we also include a parameter for the mean). Here we utilize $f_{\varphi}$ given by (1), and $\varphi$ consists of all the memory parameters, together with the ZP locations (unless these are assumed to be known based 
on metadata) and the short memory cepstral coefficients $g_{j}$. This assumes some truncation order $q$ for the EXP portion of the model. The innovations algorithm will evaluate both summands of (7), given only the acf sequence of $f_{\varphi}$. The Whittle likelihood is instead given by

$$
\mathcal{W}(\varphi ; X)=X^{\prime} \Sigma\left(f_{\varphi}^{-1}\right) X+\log \sigma^{2}
$$

where recall that $\sigma^{2}$ equals the exponential of $g_{0}$, the initial cepstral coefficient. Here we must compute the autocovariance of the inverse spectral density, but given the form of (1) this is quite easy to find: we just flip the sign on all the memory parameters $d$, as well as the cepstral coefficients $g_{j}$, and apply the algorithm above, followed by evaluation of (8). Together with a routine to conduct nonlinear optimization, either objective function can be utilized to fit these models to time series data ( $\mathrm{R}$ code is available from the authors), resulting in maximum likelihood estimates (MLEs) and Whittle likelihood estimates (WLEs) respectively. Mean effects can also be easily added if desired, either by direct optimization over specified regression effects or via a concentrated likelihood utilizing GLS formulas (cf. discussion in McElroy and Holan (2014)).

\section{Accuracy and Speed of the Algorithm}

A discussion of other methodologies is given in McElroy and Holan (2012). For a FEXP - defined as the special case of a $k$-GEXP with a single pole at frequency zero - the splitting method can be utilized to compute autocovariance. This represents a trivial case of the present algorithm. Similarly, it is known that a 1-GEXP (having a single pole, which can be located at any frequency) can be handled by the splitting method. McElroy and Holan (2012) considered efficient approximation schemes for 2-GEXP and higher order $k$-GEXP processes, for which the splitting method is not viable. (The approach of McElroy and Holan (2012) is based on truncating the infinite series representation of the autocovariance function, and utilizing special functions to approximate the truncation error.)

In order to make comparisons, it is necessary to know the true autocovariances of a $k$-GEXP. However, this is the very quantity we are trying to compute, and it is not available to arbitrary accuracy. There is available an asymptotic formula for the log determinant (ldet) of the autocovariance matrix, and so we can compare the ldet for our method, the McElroy and Holan (2012) method, and the ldet based upon the asymptotic formula - given in Proposition 1 of McElroy and Holan (2012) - denoted as $\operatorname{ldet}_{\text {Prop }}$. 


\begin{tabular}{|c|c|c|c|c|c|c|}
\hline \multicolumn{7}{|c|}{$\log |\Sigma|:$ 1-GEXP: $g=(0, .75) ; \omega=.56$} \\
\hline & \multicolumn{3}{|c|}{ ldet: $n=500$} & \multicolumn{3}{|c|}{ ldet: $n=1000$} \\
\hline$c_{1}$ & Prop & New & $\mathrm{MH}_{2012}$ & Prop & New & $\mathrm{MH}_{2012}$ \\
\hline .1 & 0.4299917 & $\begin{array}{c}.4299917 \\
(1.696498 \mathrm{e}-5)\end{array}$ & $\begin{array}{c}.429932 \\
(1.568073 \mathrm{e}-5) \\
J_{\text {Trunc }}=5,000\end{array}$ & 0.44379 & $\begin{array}{c}.4437882 \\
(9.213278 \mathrm{e}-6)\end{array}$ & $\begin{array}{c}.443785 \\
(5.240159 \mathrm{e}-6) \\
J_{\text {Trunc }}=5,000\end{array}$ \\
\hline .25 & 1.582358 & $\begin{array}{c}1.582393 \\
(3.501754 \mathrm{e}-5)\end{array}$ & $\begin{array}{c}1.582377 \\
(1.854933 \mathrm{e}-5) \\
J_{\text {Trunc }}=25,000\end{array}$ & 1.669002 & $\begin{array}{c}1.66902 \\
(1.775161 \mathrm{e}-5)\end{array}$ & $\begin{array}{c}1.668987 \\
(-1.433973 \mathrm{e}-5) \\
J_{\text {Trunc }}=25,000\end{array}$ \\
\hline .35 & 3.058414 & $\begin{array}{c}3.058385 \\
(-2.889742 \mathrm{e}-5)\end{array}$ & $\begin{array}{c}3.058331 \\
(-8.284450 \mathrm{e}-5) \\
J_{\text {Trunc }}=100,000\end{array}$ & 3.228235 & $\begin{array}{c}3.228223 \\
(-1.230519 \mathrm{e}-5)\end{array}$ & $\begin{array}{c}3.228115 \\
(-1.201864 \mathrm{e}-4) \\
J_{\text {Trunc }}=100,000\end{array}$ \\
\hline .45 & 5.973976 & $\begin{array}{c}5.973767 \\
(-2.088053 \mathrm{e}-4)\end{array}$ & $\begin{array}{c}5.972568 \\
(-1.407956 \mathrm{e}-3) \\
J_{\text {Trunc }}=100,000\end{array}$ & 6.254700 & $\begin{array}{c}6.2546 \\
(-9.965885 \mathrm{e}-5)\end{array}$ & $\begin{array}{c}6.252199 \\
(-2.501926 \mathrm{e}-3) \\
J_{\text {Trunc }}=100,000\end{array}$ \\
\hline
\end{tabular}

Table 1: Log determinant of the autocovariance sequence for a 1-GEXP model obtained using the asymptotic approximation of Proposition 1 of McElroy and Holan (2012) (Prop), the algorithm of Section 3 (New), and the truncation approach of McElroy and Holan(2012) $\left(\mathrm{MH}_{2012}\right)$. In the case of the $\mathrm{MH}_{2012}$ approach we provide the truncation used, $J_{\text {Trunc }}$. The number in parenthesis denotes the difference between the estimated ldet and $\operatorname{ldet}_{\text {Prop }}$. In the case of $\mathrm{MH}_{2012}, J_{\text {Trunc }}$ was chosen to reflect the smallest difference between $\operatorname{ldet}_{\text {Prop }}$ and the ldet from $\mathrm{MH}_{2012}$.

In the case of fractional Gaussian noise the ldet of the autocovariance sequence can be exactly calculated or approximated to any degree of accuracy using the splitting method. In this case, examining the ldet of the newly proposed approach, we find that all estimated ldets are within 1e-4 of ldet $_{\text {Prop }}$ and within 1e-12 of the ldet obtained using splitting method - even for $d=.45$ (for both $n=500$ and 1000). Similarly, for FEXP processes with unit innovation variance and various short memory specifications, all estimated ldets are within 1e-4 of the asymptotic ldet (ldet ${ }_{\text {Prop }}$ ) and within 1e-12 of the ldet obtained using splitting method - even for $d=.45$ (for both $n=500$ and 1000).

For the 1-GEXP case, we consider sample sizes of $n=500$ and 1000 with long memory parameter $c_{1}$ ranging from .1 to .45. Additionally, the seasonal frequency is set at $\omega=.56$, with the short memory portion $g=\left(g_{0}, g_{1}\right)=(0, .75)$ - which implies unit innovation variance. In particular, we compare the difference between the ldet of the new approach and the asymptotic ldet to the difference between the ldet from the truncation approach and the asymptotic ldet (Table 1). Note that, for the method of McElroy and Holan (2012), the truncation level $J_{\text {Trunc }}$ was set between 5,000 and 100,000 .

From Table 1, we see that both approaches are highly accurate and closely agree with the 
asymptotic approximation. Further, for the 1-pole case, the number of terms needed for accurate approximation increases as the degree of persistence increases. Even for the case of $c_{1}=.25$, it happens that 25,000 terms (Wold coefficients) are needed in order to obtain an accurate approximation (for both $n=500$ and 1000). The number of terms quickly increases to 100,000 for $c_{1}=.35$. Consequently, computation is significantly more expensive for the truncation method relative to the newly proposed algorithm.

The 2-GEXP case presents additional complications, as there are now multiple seasonal poles in the spectral density. Here, we consider sample sizes of $n=500$ and 1000 with long memory parameters $\left(c_{1}, c_{2}\right)$ ranging between $(.1, .2)$ to $(.1, .45)$ and $(.45, .2)$ to $(.45, .4)$. Additionally, the seasonal frequency is set at $\omega=(.1, .56)$, with the short memory portion $g=\left(g_{0}, g_{1}\right)=(0, .75)$ (implying unit innovation variance). Again, we compare the difference between the ldet of the new approach and the asymptotic ldet to the difference between the ldet from the truncation approach and the asymptotic ldet (Table 2).

From Table 2, we see that both approaches are fairly accurate and are in agreement with the asymptotic approximation. However, in contrast to the 1-GEXP case, there is no definitive correspondence between the truncation needed for the approach of McElroy and Holan (2012) $\left(\mathrm{MH}_{2012}\right)$ and the degree of cyclical persistence; i.e., the truncation point in the $\mathrm{MH}_{2012}$ approach is a non-monotonic function of the level of cyclical persistency. Also, although for moderately persistent cases the $\mathrm{MH}_{2012}$ method is a slightly better appproximation to $\operatorname{ldet}_{\mathrm{Prop}}$, this behavior is highly dependent on the truncation chosen, which would be unknown in practice. This correspondence may be due to the fact that the McElroy and Holan (2012) method is based upon asymptotic approximations, thereby generating a closer association to the asymptotic $\operatorname{ldet}_{\text {Prop }}$.

Aside from accuracy, the main advantages of the new algorithm are computational efficiency and the ability to easily accommodate spectra with more than two poles. The capacity to readily compute the autocovariance sequence in the multiple-pole setting is extremely advantageous and not feasible without the algorithm of Section 3 . In terms of speed, we compare the computation time of one likelihood evaluation for several cases. In particular, we compute the likelihood with the autocovariance matrix calculated using the truncation approach of McElroy and Holan (2012) and using the new algorithm. All computations are carried out in the $\mathrm{R}$ programming language (R Development Core Team, 2014) using a MacBook Pro 2.7 GHz Intel Core i7 with 16 GB 1600 $\mathrm{MHz}$ DDR3 RAM.

The first example considers the 2-GEXP specified in Table 2 with $\left(c_{1}, c_{2}\right)=(.1, .45)$. For $n=500$ 


\begin{tabular}{|c|c|c|c|c|c|c|}
\hline \multicolumn{7}{|c|}{$\log |\Sigma|:$ 2-GEXP: $g=(0, .75) ; \omega=(.1, .56)$} \\
\hline & \multicolumn{3}{|c|}{ ldet: $n=500$} & \multicolumn{3}{|c|}{ ldet: $n=1000$} \\
\hline$\left(c_{1}, c_{2}\right)$ & Prop & New & $\mathrm{MH}_{2012}$ & Prop & New & $\mathrm{MH}_{2012}$ \\
\hline$(.1, .2)$ & 1.528072 & $\begin{array}{c}1.497591 \\
(-3.048125 \mathrm{e}-2)\end{array}$ & $\begin{array}{c}1.527665 \\
(-4.072432 \mathrm{e}-4) \\
J_{\text {Trunc }}=100,000\end{array}$ & 1.597387 & $\begin{array}{c}1.570535 \\
(-2.685184 \mathrm{e}-2)\end{array}$ & $\begin{array}{c}1.596123 \\
(-1.263973 \mathrm{e}-3) \\
J_{\text {Trunc }}=50,000\end{array}$ \\
\hline$(.1, .3)$ & 2.714834 & $\begin{array}{c}2.687255 \\
(-2.757927 \mathrm{e}-2)\end{array}$ & $\begin{array}{c}2.714331 \\
(-5.028845 \mathrm{e}-4) \\
J_{\text {Trunc }}=100,000\end{array}$ & 2.853464 & $\begin{array}{c}2.830055 \\
(-2.340929 \mathrm{e}-2)\end{array}$ & $\begin{array}{c}2.852651 \\
(-8.126799 \mathrm{e}-4) \\
J_{\text {Trunc }}=100,000\end{array}$ \\
\hline$(.1, .45)$ & 6.538299 & $\begin{array}{c}6.515872 \\
(-2.242651 \mathrm{e}-2)\end{array}$ & $\begin{array}{c}6.535380 \\
(-2.918457 \mathrm{e}-4) \\
J_{\text {Trunc }}=100,000\end{array}$ & 6.832886 & $\begin{array}{c}6.811977 \\
(-2.090914 \mathrm{e}-2)\end{array}$ & $\begin{array}{c}6.834062 \\
(1.175967 \mathrm{e}-3) \\
J_{\text {Trunc }}=50,000\end{array}$ \\
\hline$(.45, .2)$ & 8.134616 & $\begin{array}{c}8.093399 \\
(-4.121709 \mathrm{e}-2)\end{array}$ & $\begin{array}{c}8.13965 \\
(5.0335 \mathrm{e}-3) \\
J_{\text {Trunc }}=50,000\end{array}$ & 8.470792 & $\begin{array}{c}8.437261 \\
(-3.353121 \mathrm{e}-2)\end{array}$ & $\begin{array}{c}8.486669 \\
(1.587655 \mathrm{e}-2) \\
J_{\text {Trunc }}=50,000\end{array}$ \\
\hline$(.45, .3)$ & 9.492050 & $\begin{array}{c}9.457665 \\
(-3.438547 \mathrm{e}-2)\end{array}$ & $\begin{array}{c}9.494429 \\
(2.378529 \mathrm{e}-3) \\
J_{\text {Trunc }}=25,000\end{array}$ & 9.897542 & $\begin{array}{c}9.874612 \\
(-2.292996 \mathrm{e}-2)\end{array}$ & $\begin{array}{c}9.856134 \\
(-4.140792 \mathrm{e}-2) \\
J_{\text {Trunc }}=50,000\end{array}$ \\
\hline$(.45, .4)$ & 11.682510 & $\begin{array}{c}11.66138 \\
(-2.113188 \mathrm{e}-2)\end{array}$ & $\begin{array}{c}11.273010 \\
(-0.409498) \\
J_{\text {Trunc }}=50,000\end{array}$ & 12.185040 & $\begin{array}{c}12.173 \\
(-1.200251 \mathrm{e}-2)\end{array}$ & $\begin{array}{c}11.807010 \\
(-0.378036) \\
J_{\text {Trunc }}=25,000\end{array}$ \\
\hline
\end{tabular}

Table 2: Log determinant of the autocovariance sequence for a 2-GEXP model obtained using the asymptotic approximation of Proposition 1 of McElroy and Holan (2012) (Prop), the algorithm of Section 3 (New), and the truncation approach of McElroy and Holan(2012) $\left(\mathrm{MH}_{2012}\right)$. In the case of the $\mathrm{MH}_{2012}$ approach we provide the truncation used, $J_{\text {Trunc }}$. The number in parenthesis denotes the difference between the estimated ldet and ldet $t_{\text {Prop }}$. In the case of $\mathrm{MH}_{2012}, J_{\text {Trunc }}$ was chosen to reflect the smallest difference between $\operatorname{ldet}_{\text {Prop }}$ and the ldet from $\mathrm{MH}_{2012}$. 
$\left(J_{\text {Trunc }}=100,000\right)$, the computation time for one likelihood evaluation of the $\mathrm{MH}_{2012}$ approach is 3.074 minutes, whereas the new algorithm (with mesh size 10001) is 1.180 seconds. Similarly, for $n=1000\left(J_{\text {Trunc }}=50,000\right)$, the $\mathrm{MH}_{2012}$ approach takes 51.410 seconds for one likelihood evaluation, whereas the new algorithm took only 2.478 seconds (with mesh size 10001). For $\left(c_{1}, c_{2}\right)=(.45, .4)$, with $n=500\left(J_{\text {Trunc }}=50,000\right)$ the $\mathrm{MH}_{2012}$ computation time for one likelihood evaluation was 50.167 seconds, whereas with the new approach the computation took 1.229 seconds. Finally for $\left(c_{1}, c_{2}\right)=(.45, .4)$, with $n=1000\left(J_{\text {Trunc }}=25,000\right)$ the $\mathrm{MH}_{2012}$ computation time was 15.275 seconds for one likelihood evaluation, whereas with the new approach the computation took 2.548 seconds.

It is important to emphasize that all computations done here are completely in $\mathrm{R}$ ( $\mathrm{R}$ Development Core Team, 2014). In order to make estimation feasible, McElroy and Holan (2012) made use of Fortran subroutines using the $\mathrm{R}$ package inline. Without the use of a lower level programming language such as Fortran or $\mathrm{C}++$, maximum likelihood estimation would be impractical using the approach of McElroy and Holan (2012). In contrast, the new approach easily handles such computations without the need to "optimize" computer code.

\section{Case Studies}

To illustrate the utility of the algorithm introduced in Section 3 , we examine four different datasets. The four examples demonstrate the ability to compute autocovariances in our new approach under various model specifications (i.e., varying the number of long range persistencies). In only the first example is another method viable, because $k$ is small $(k=2)$; the other examples have $k=7$ or $k=27$ poles, so that only our proposed method is feasible.

\subsection{Quasi-biennial Oscillations}

We consider a series consisting of 11, 000 daily observations beginning November 19, 1964, corresponding to the east/west wind component from Truk Island (see Cressie and Wikle, 2011, Chapter 3), which are initially downsampled at a rate of 50 days in order to facilitate computation. Our chief interest is in determining the exact period of the secondary spectral peak (corresponding to a period of roughly 2.3 years). This latter effect is referred to as a quasi-biennial oscillation (QBO), and is further described in Andrews et al. (1987) and Wikle et al. (1995); Figure 1 displays the data (before downsampling) along with the log spectrum (cepstrum) from fitted models. 


\begin{tabular}{|c|c|c|c|c|}
\hline \multicolumn{5}{|c|}{ downsample rate 50} \\
\hline & \multicolumn{2}{|c|}{ MLE } & \multicolumn{2}{c|}{ WLE } \\
\hline Parameter & Est & s.e. & Est & s.e. \\
\hline$g_{1}$ & -2.025 & .328 & -2.019 & .328 \\
$g_{2}$ & -.389 & .170 & -.391 & .170 \\
$g_{3}$ & .230 & .175 & .229 & .175 \\
$g_{4}$ & .021 & .158 & .020 & .158 \\
$g_{5}$ & -.255 & .141 & -.254 & .141 \\
$\omega_{1}$ & $2.033(2.387)$ & .522 & $2.036(2.387)$ & .522 \\
$\omega_{2}$ & $1.841(1.003)$ & .590 & $1.843(1.003)$ & .590 \\
$c_{1}$ & $-2.043(.384)$ & .015 & $-2.053(.385)$ & .015 \\
$c_{2}$ & $-1.523(.363)$ & .015 & $-1.516(.363)$ & .015 \\
$\mu$ & -4.551 & .273 & -4.552 & .273 \\
\hline
\end{tabular}

Table 3: Parameter estimates for the 2-GEXP(5) fitted to the QBO data, downsampled at rate 50 . The peak frequencies (period in years in parentheses) correspond to $\omega_{1}$ and $\omega_{2}$, while the peak strengths are $c_{1}$ and $c_{2}$ (values mapped to $(-1 / 2,1 / 2)$ given in parentheses).

We then fitted a 2-GEXP(5) model with two seasonal poles to the series. The results are given in Table 3, for MLEs and WLEs with their standard errors. Note that we parametrize the peak locations and persistencies with real numbers, which are then mapped into bounded intervals this ensures that peak persistencies take values in $(-1 / 2,1 / 2)$. The standard errors (obtained from the numerical Hessians) are for these original parameters, but in parentheses are the transformed values.

We observe that the chief periods are 2.387 years and 1.003 years, with respective persistencies .384 and .363, corresponding to the QBO and annual effect. In this case, the results for the Gaussian likelihood and the Whittle were extremely similar, with standard errors the same up to three decimal places accuracy. In order to examine the impact of downsampling, we changed the rate to 25 days and re-ran the results - with a lower downsampling rate, the effective sample size increases but the two spectral peaks will appear closer together in spectral estimates. Table 4 gives these results, which yield essentially the same periods (though the peak strengths are different). It is interesting that the standard errors were also the same as those obtained with a higher downsampling rate.

\subsection{Industrial Production}

The monthly series of Industrial Production (Federal Reserve Board; http://research.stlouisfed. org/fred2/) is available from 1919 onwards. Having first adjusted the data for a trading day effect, Easter holiday effects, and additive outliers, we then studied the years 1949.1 through 2007.12 on 


\begin{tabular}{|c|c|c|c|c|}
\hline \multicolumn{5}{|c|}{ downsample rate 25 } \\
\hline & \multicolumn{2}{|c|}{ MLE } & \multicolumn{2}{c|}{ WLE } \\
\hline Parameter & Est & s.e. & Est & s.e. \\
\hline$g_{1}$ & -2.422 & .328 & -2.422 & .328 \\
$g_{2}$ & -.986 & .170 & -.986 & .170 \\
$g_{3}$ & -.381 & .175 & -.381 & .175 \\
$g_{4}$ & -.330 & .158 & -.330 & .158 \\
$g_{5}$ & -.105 & .141 & -.105 & .141 \\
$\omega_{1}$ & $3.045(2.386)$ & .522 & $3.045(2.386)$ & .522 \\
$\omega_{2}$ & $1.499(1.005)$ & .590 & $1.499(1.005)$ & .590 \\
$c_{1}$ & $-2.798(.455)$ & .015 & $-2.798(.455)$ & .015 \\
$c_{2}$ & $-2.393(.317)$ & .015 & $-2.393(.317)$ & .015 \\
$\mu$ & -4.926 & .273 & -4.930 & .273 \\
\hline
\end{tabular}

Table 4: Parameter estimates for the 2-GEXP(5) fitted to the QBO data, downsampled at rate 25 . The peak frequencies (period in years in parentheses) correspond to $\omega_{1}$ and $\omega_{2}$, while the peak strengths are $c_{1}$ and $c_{2}$ (values mapped to $(-1 / 2,1 / 2)$ given in parentheses).
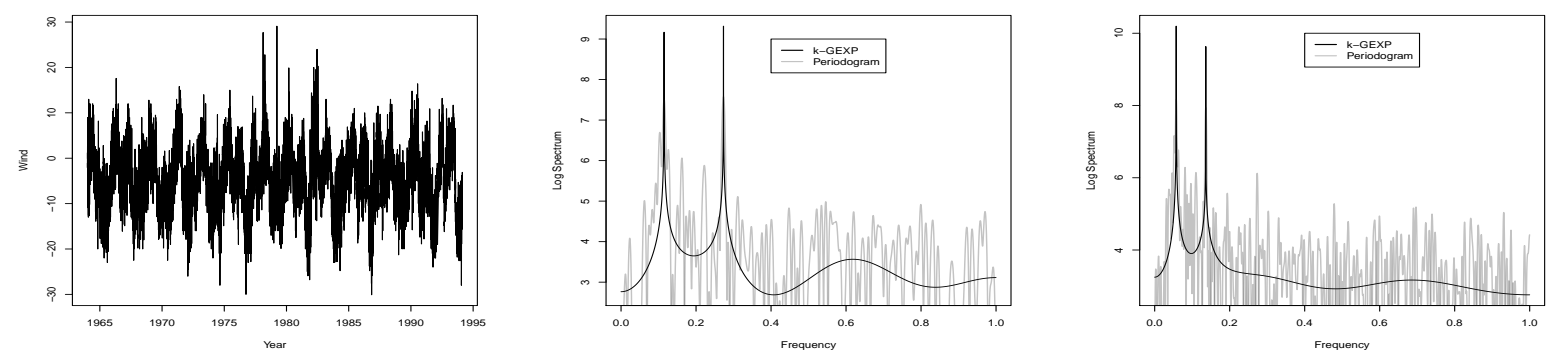

Figure 1: Plot of 11,000 observations of the east/west wind component from Truk Island beginning November 19, 1964 (left panel) and estimated cepstral representation (log spectrum) (central and right panels), based on the 2-GEXP model fitted to the downsampled data (at a rate of 50 and 25 days) via maximum likelihood estimation, with the log periodogram (bias-adjusted) overlaid.

the log-scale. Nonstationary trend effects in the data required a single differencing; although a seasonal difference could be applied as well, we attempt estimation of the seasonal poles through a GEXP model instead. Although the seasonal amplitude is small and hard to discern (left panel of Figure 2), the data does indeed have terrestrial periodicities, being sampled at monthly intervals, and hence we expect the spectral peaks to have the form $2 \pi j / 12$. Therefore, the differenced $\log$ series was fitted by a 7 -GEXP(3) model where the trend/seasonal poles were fixed at $\pi j / 6$ for $0 \leq j \leq 6$. We might expect $a, b \neq 0$ in the model, and $c_{j} \neq 0$ for $1 \leq j \leq 5$, which is indeed borne out by our estimates. As previously discussed, the choice of the order for the short memory EXP portion was only meant for illustration and could be formally chosen through model selection methods if desired. 


\begin{tabular}{|c|c|c|c|c|}
\hline & \multicolumn{2}{|c|}{ MLE } & \multicolumn{2}{c|}{ WLE } \\
\hline Parameter & Est & s.e. & Est & s.e. \\
\hline$g_{1}$ & 2.984 & .415 & 2.997 & .328 \\
$g_{2}$ & 1.318 & .164 & 1.304 & .148 \\
$g_{3}$ & .528 & .117 & .505 & .111 \\
$a$ & $-.800(-.190)$ & .416 & $-.571(-.139)$ & .374 \\
$c_{1}$ & $.490(.121)$ & .316 & $.092(.023)$ & .202 \\
$c_{2}$ & $3.488(.470)$ & 1.258 & $3.746(.477)$ & 1.228 \\
$c_{3}$ & $4.456(.489)$ & 1.085 & $4.686(.491)$ & 1.314 \\
$c_{4}$ & $4.275(.486)$ & 1.286 & $4.427(.488)$ & 1.353 \\
$c_{5}$ & $3.489(.470)$ & .607 & $3.922(.481)$ & .943 \\
$b$ & $3.360(.467)$ & .711 & $3.722(.476)$ & 1.045 \\
$\mu$ & .003 & .0003 & .003 & .0003 \\
\hline
\end{tabular}

Table 5: Parameter estimates for the 7-GEXP(3) fitted to the Industrial Production data. The peak persistencies are $a, b$, and the $c_{j}$ (values mapped to $(-1 / 2,1 / 2)$ given in parentheses).

The results of Table 5 indicate that the trend memory is fairly weak (differencing having removed most of the structure). Moreover, for the Gaussain likelihood results the first seasonal peak is somewhat weak, but the memory at peaks two through six all exceed .45, yielding pronounced effects. For the Whittle, the point estimate for the first seasonal peak is not significantly different from short memory, whereas the results for the other peaks resemble those from the Gaussian likelihood. While the MLE and WLE estimates are broadly similar, the standard errors are quite different for some of the parameters. See Figure 2 for a plot of the observed time series and estimated (MLE) cepstral representation.

\subsection{Mauna Loa}

The Mauna Loa time series has now been studied in several papers that treat seasonal long memory. The dataset consists of 382 monthly atmospheric $\mathrm{CO}_{2}$ measurements collected at the summit of Mauna Loa in Hawaii, beginning in March 1958 (Keeling et al, 1989). While twin-peak models have been considered before for the second-differenced data, such as the 2-factor GARMA of Woodward et al. (1998) and the 2-GEXP(4) of McElroy and Holan (2012), the periodogram of the data indicates that a model accommodating additional peaks is warranted. Because the data is terrestrial and monthly, it is plausible that the spectral peak frequencies take the form $\pi j / 6$ for $1 \leq j \leq 6$, as with the previous example. Therefore we again consider the 7-GEXP(3) model, and we compute the MLEs and WLEs for the once-differenced data (which was adjusted for missing values in a 

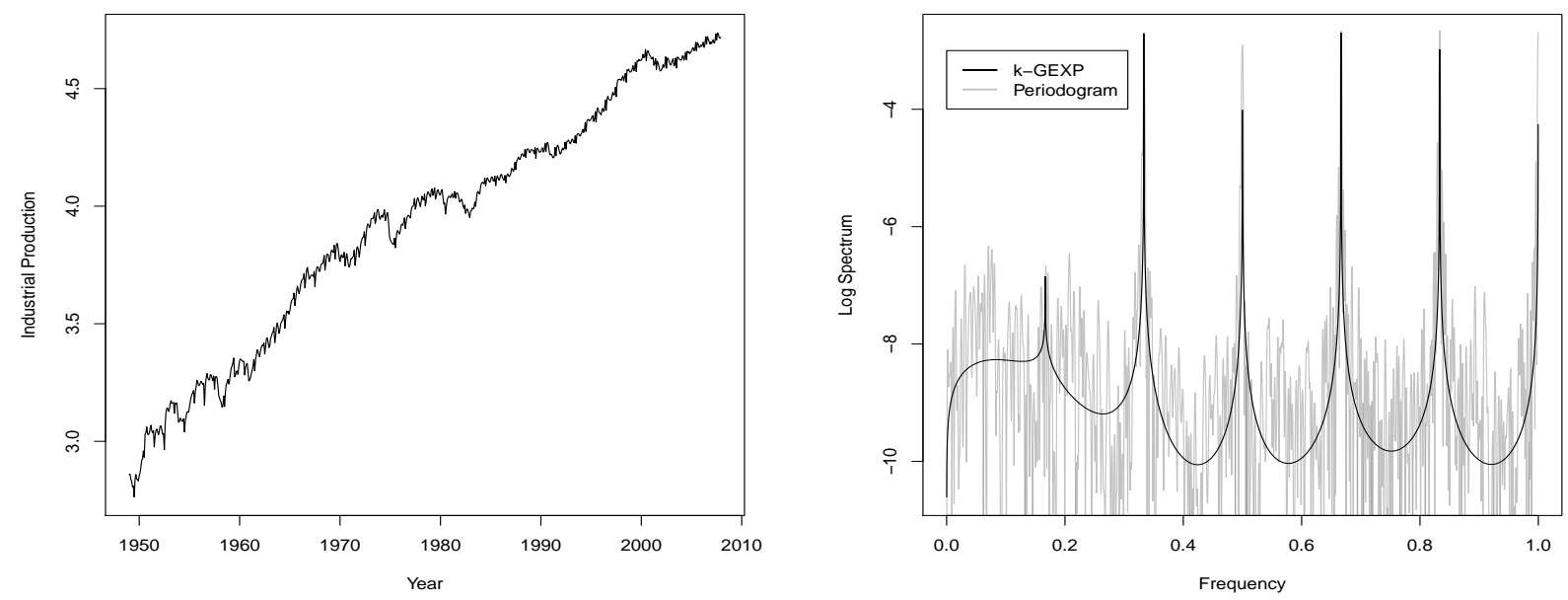

Figure 2: Plot of Industrial Production data from January 1949 through December 2007 (left panel) and estimated cepstral representation (log spectrum) (right panel), based on the 7-GEXP model fitted via maximum likelihood estimation, with the log periodogram (bias-adjusted) overlaid.

pre-processing stage).

The MLE results indicate a strong trend effect, as well as strong seasonality in the first through fourth seasonal frequencies. The point estimates for the fifth and sixth seasonal frequencies are not significantly different from short memory. Results for the WLEs are broadly similar, although the trend memory is now significantly negative; also, the standard errors for many of the parameters are smaller. See Figure 3 for a plot of the observed time series and estimated (MLE) cepstral representation.

\subsection{Unemployment Insurance Claims}

The weekly time series of unemployment insurance claims from the Bureau of Labor Statistics (1987 through 2007, for a total of 1040 observations; http://www.oui.doleta.gov/unemploy/claims) is clearly seasonal, and is a fairly important series for understanding movements in the employment sector of the economy. Like the Industrial Production example, the spectral peak frequencies will be taken as known, where $p=52$ is the number of seasons. Therefore, we assume that spectral peaks are located at frequencies $\pi j / 26$ for $1 \leq j \leq 25$, in addition to peaks at frequency 0 and $\pi$. Hence we fit a 27-GEXP(5) model via the Gaussian and Whittle likelihoods. There are 32 parameters in the model, so that the ratio of sample size to parameters is over thirty. The calculation of autocovariances for such a process is now feasible, which we illustrate through providing model- 


\begin{tabular}{|c|c|c|c|c|}
\hline & \multicolumn{2}{|c|}{ MLE } & \multicolumn{2}{c|}{ WLE } \\
\hline Parameter & Est & s.e. & Est & s.e. \\
\hline$g_{1}$ & -3.347 & .592 & -1.388 & .400 \\
$g_{2}$ & .578 & .289 & 1.446 & .223 \\
$g_{3}$ & -.394 & .189 & -.470 & .158 \\
$a$ & $1.564(.327)$ & .682 & $-.582(-.142)$ & .249 \\
$c_{1}$ & $6.476(.498)$ & 1.004 & $6.951(.499)$ & 1.995 \\
$c_{2}$ & $4.402(.488)$ & .934 & $4.808(.492)$ & 1.929 \\
$c_{3}$ & $1.924(.373)$ & .503 & $1.805(.359)$ & .426 \\
$c_{4}$ & $1.482(.315)$ & .416 & $2.233(.403)$ & .748 \\
$c_{5}$ & $.133(.033)$ & .317 & $.126(.031)$ & .219 \\
$b$ & $-.223(-.055)$ & .643 & $-.487(-.119)$ & .433 \\
$\mu$ & .101 & .025 & .099 & .006 \\
\hline
\end{tabular}

Table 6: Parameter estimates for the 7-GEXP(3) fitted to the Mauna Loa data. The peak persistencies are $a, b$, and the $c_{j}$ (values mapped to $(-1 / 2,1 / 2)$ given in parentheses).

fitting results.

The MLE and WLE estimates are broadly similar, although the standard errors are different in some cases. Excepting the trend frequency, all of the spectral peak parameters are positive, and some are quite large, indicating substantial persistence. It is interesting that one of the weakest peaks is the thirteenth (.171), which corresponds to quarterly phenomena, whereas the first seasonal peak (.452), which corresponds to once-a-year phenomena, is one of the strongest. See Figure 4 for a plot of the observed time series and estimated (MLE) cepstral representation.

\section{Discussion}

This paper presents a new algorithm, that allows for computation of the autocovariance function of time series models with multiple persistencies. This is accomplished by rewriting the model spectral density as a sum of single pole factors, and using the fast splitting method on each summand. Our numerical work shows the accuracy of this approach, and the gains in computation time over previous methods are substantial. More importantly, the encoding for multiple peak processes is straightforward, compared to the algorithm of McElroy and Holan (2012).

Our focus is not on modeling, although four case studies are provided in order to illustrate that likelihood evaluation is feasible. We have used the $k$-GEXP model to demonstrate the new algorithm, but $k$-GARMA variants could also be devised along the same lines, with similar gains to speed and accuracy. Previous analysis of time series with multiple persistencies has been hampered 


\begin{tabular}{|c|c|c|c|c|}
\hline & \multicolumn{2}{|l|}{ MLE } & \multicolumn{2}{|l|}{ WLE } \\
\hline Parameter & Est & s.e. & Est & s.e. \\
\hline$g_{1}$ & -1.672 & 1.104 & -1.559 & 1.810 \\
\hline$g_{2}$ & -1.385 & .591 & -1.284 & .887 \\
\hline$g_{3}$ & -.282 & .429 & -.231 & .785 \\
\hline$g_{4}$ & .369 & .339 & .388 & .558 \\
\hline$g_{5}$ & -.040 & .296 & -.040 & .478 \\
\hline$a$ & $-.014(-.004)$ & .363 & $.014(.003)$ & .495 \\
\hline$c_{1}$ & $2.978(.452)$ & .769 & $2.802(.443)$ & 1.580 \\
\hline$c_{2}$ & $4.089(.484)$ & .829 & $4.225(.486)$ & 1.325 \\
\hline$c_{3}$ & $2.603(.431)$ & .588 & $2.703(.437)$ & 1.388 \\
\hline$c_{4}$ & $3.489(.470)$ & .758 & $3.667(.475)$ & 1.267 \\
\hline$c_{5}$ & $2.000(.381)$ & .425 & $2.083(.389)$ & 1.319 \\
\hline$c_{6}$ & $2.165(.397)$ & .556 & $1.931(.373)$ & 1.107 \\
\hline$c_{7}$ & $.856(.202)$ & .320 & $.888(.208)$ & .605 \\
\hline$c_{8}$ & $2.489(.423)$ & .666 & $2.254(.405)$ & 1.193 \\
\hline$c_{9}$ & $1.342(.293)$ & .332 & $1.371(.298)$ & .652 \\
\hline$c_{10}$ & $1.093(.249)$ & .321 & $1.103(.251)$ & .584 \\
\hline$c_{11}$ & $1.706(.346)$ & .533 & $1.639(.337)$ & .941 \\
\hline$c_{12}$ & $3.956(.481)$ & .992 & $4.268(.486)$ & 1.708 \\
\hline$c_{13}$ & $.712(.171)$ & .303 & $.698(.168)$ & .436 \\
\hline$c_{14}$ & $1.047(.240)$ & .362 & $1.034(.238)$ & .570 \\
\hline$c_{15}$ & $.896(.210)$ & .372 & $.866(.204)$ & .503 \\
\hline$c_{16}$ & $2.351(.413)$ & .881 & $2.432(.419)$ & 1.611 \\
\hline$c_{17}$ & $.943(.220)$ & .376 & $.898(.210)$ & .489 \\
\hline$c_{18}$ & $3.562(.472)$ & 1.201 & $3.506(.471)$ & 1.431 \\
\hline$c_{19}$ & $2.607(.431)$ & 1.048 & $2.550(.428)$ & 1.018 \\
\hline$c_{20}$ & $1.499(.317)$ & .488 & $1.436(.308)$ & .557 \\
\hline$c_{21}$ & $1.139(.258)$ & .429 & $1.120(.254)$ & .537 \\
\hline$c_{22}$ & $3.033(.454)$ & .975 & $3.096(.457)$ & 1.095 \\
\hline$c_{23}$ & $1.550(.325)$ & .494 & $1.508(.319)$ & .576 \\
\hline$c_{24}$ & $3.473(.470)$ & .954 & $3.666(.475)$ & 1.247 \\
\hline$c_{25}$ & $1.526(.321)$ & .555 & $1.481(.315)$ & .626 \\
\hline$b$ & $1.790(.357)$ & .702 & $1.744(.351)$ & .826 \\
\hline$\mu$ & -.00074 & .0015 & -.00088 & .0029 \\
\hline
\end{tabular}

Table 7: Parameter estimates for the 27-GEXP(5) fitted to the Unemployment Insurance Claims data. The peak persistencies are $a, b$, and the $c_{j}$ (values mapped to $(-1 / 2,1 / 2)$ given in parentheses). 

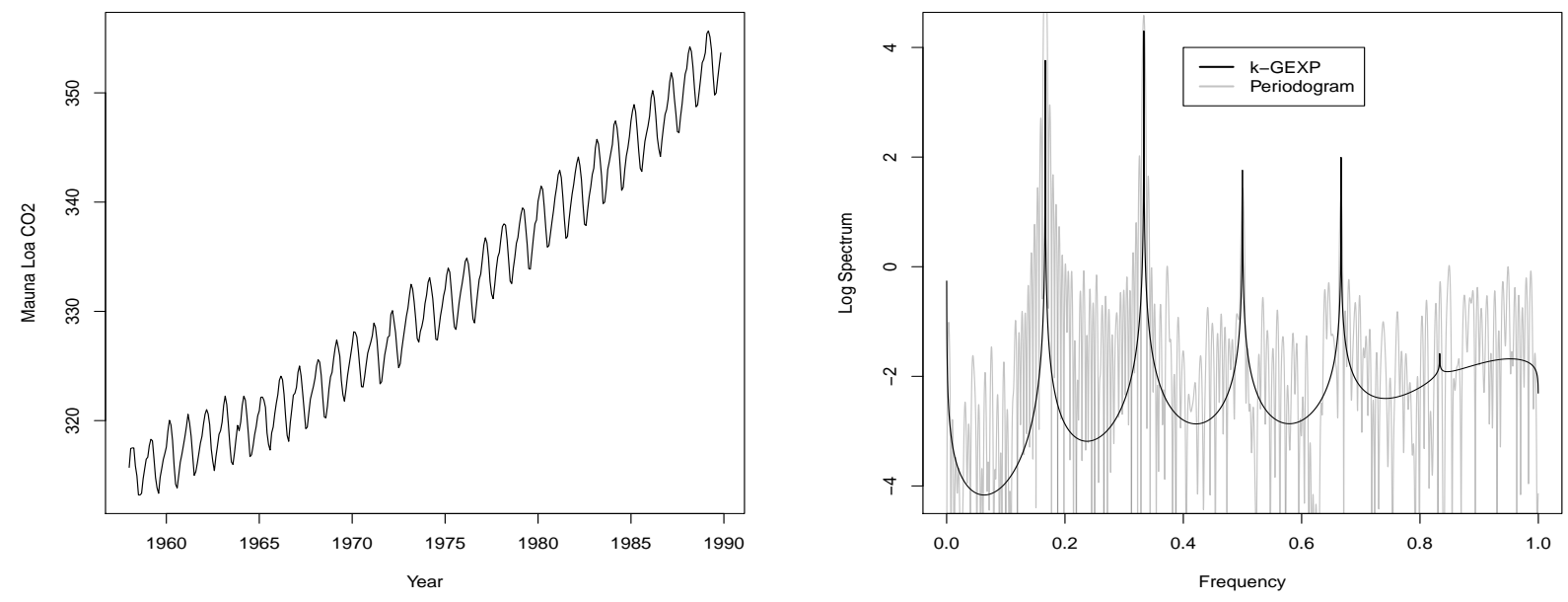

Figure 3: Plot of Mauna Loa $\mathrm{CO}_{2}$ data for 358 observations beginning March 1958 (left panel) and estimated cepstral representation (log spectrum) for the first-differenced time series (right panel), based on the 7-GEXP model fitted via maximum likelihood estimation, with the log periodogram (bias-adjusted) overlaid.

by computation time; this work enables practitioners to more readily utilize multiple-pole models.

\section{Acknowledgments}

The authors thank Christopher Wikle for suggesting and providing the dataset used in Section 5.1 and for useful discussion. This research was partially supported by the U.S. National Science Foundation (NSF) and the U.S. Census Bureau under NSF grant SES-1132031, funded through the NSF-Census Research Network (NCRN) program. Finally, we thank the Associate Editor, and two anonymous Referees for providing valuable comments that helped strengthen this manuscript.

\section{Appendix}

Proof of Proposition 1. Because $f=f^{-} f^{+} g$, it suffices to prove that $f_{Z P}=f^{+} \cdot \nu$. Using $d=d^{+}+d^{-}$for all values of $d$, and multiplying each term of $\nu$ by $f^{+}$, we obtain $f_{Z P}$ at once. The assertions about $\nu$ follow because the function evidently has no poles, and being a sum of non-negative terms can only have a zero if this zero is common to all the summands; but this is impossible due to the distinct locations of the various $\vartheta$ frequencies. (In the case where there is only one ZP factor, $\nu$ would indeed have a zero.) 

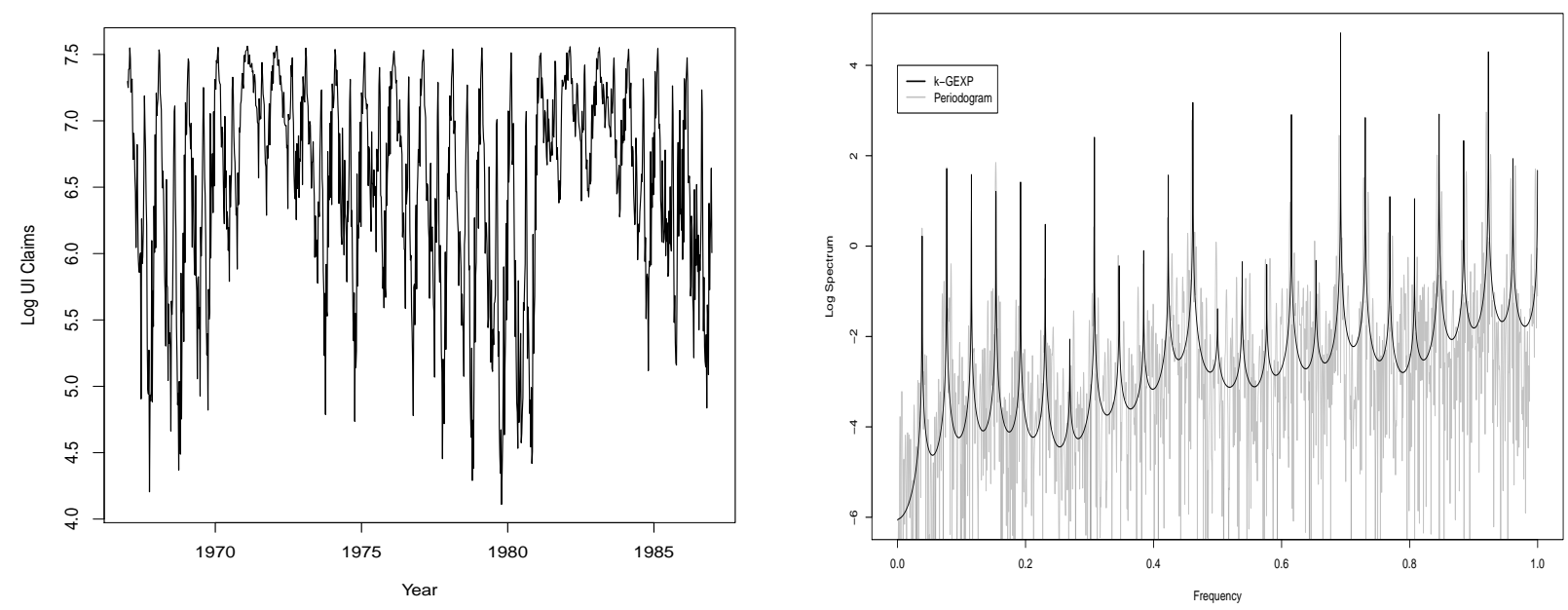

Figure 4: Plot of log unemployment insurance claims data from January 1987 through December 2007 (left panel) and estimated cepstral representation (log spectrum) (right panel), based on the 27GEXP model fitted via maximum likelihood estimation, with the log periodogram (bias-adjusted) overlaid.

Proof of Proposition 2, The first formula is straight convolution, while the second formula for the ZP factor follows from the $\operatorname{ARFIMA}(0, d, 0)$ results described in Brockwell and Davis (1991) in the cases that $\vartheta=0, \pi$. For the third case, we refer to the $\operatorname{GARMA}(0, d, 0)$ results of Chung (1996).

\section{References}

[1] Andrews, D.G., Holton, J.R., and Leovy, C.B. (1987). Middle Atmospheric Dynamics. Academic Press.

[2] Beran, J. (2010). Long-range dependence. WIREs Comput. Stat. 2, 26-35.

[3] Bertelli, S. and Caporin, M. (2002). A note on calculating autocovariances of long-memory processes. J. Time Series Analysis 23, 503-508.

[4] Bisaglia, L., Bordignon, S. and Lisi, F. (2003). k-Factor GARMA models for intraday volatility forecasting. Appl. Econom. Lett. 10, 251-254.

[5] Bloomfield, P. (1973). An exponential model for the spectrum of a scalar time series. Biometrika 60, 217-226.

[6] Brockwell, P. and Davis, R. (1991). Time Series: Theory and Methods New York: Springer. 
[7] Cressie, N. and Wikle, C.K. (2011). Statistics for Spatio-Temporal Data, Hoboken, NJ: Wiley.

[8] Chung, C. (1996). Estimating a generalized long memory process. Journal of Econometrics $73,237-259$.

[9] Ferrara, L. and Guégan, D. (2000). Forecasting financial time series with generalized long memory processes. In Advances in Quantitative Asset Management (Edited by C. L. Dunis), 319-342. Kluwer Academic Publishers.

[10] Gil-Alana, L. A. (2008). Cyclical long-range dependence and the warming effect in a long temperature time series. Internat. J. Climatology 28, 1435-1443.

[11] Gray, H., Zhang, N. and Woodward, W. (1989). On Generalized Fractional Processes. J. Time Ser. Anal. 10, 233-257.

[12] Holan, S. and McElroy, T. (2012). On the seasonal adjustment of long memory time series," in Economic Time Series: Modeling and Seasonality, eds. W. Bell, S. Holan, and T. McElroy, New York: Chapman and Hall.

[13] Hosking, J. (1981). Fractional differencing. Biometrika 68, 165-176.

[14] Hurvich, C. (2002). Multistep forecasting of long memory series using fractional exponential models. Internat. J. Forecasting 18, 167-179.

[15] Keeling, C.D., Bacastow, R.B., Carter, A.F., Piper, S.C. and Whorf, T.P. (1989). Aspects of climate variability in the Pacific and the Western Americas. Am. Geophys. Union, Gephys. Monogr. 55, 165-236.

[16] McElroy, T. and Holan, S. (2012). On the estimation of autocovariances for generalized Gegenbauer processes. Statistica Sinica 22, 1661-1687.

[17] McElroy, T. and Holan, S. (2014). Asymptotic theory of cepstral random fields. Annals of Statistics 42, 64-86.

[18] McElroy, T. and Politis, D. (2014). Spectral density and spectral distribution inference for long memory time series via fixed-b asymptotics. Journal of Econometrics 182, 211-225.

[19] Porter-Hudak, S. (1990). An application of the seasonal fractionally differenced model to the monetary aggregates. J. Amer. Statist. Assoc. 85, 338-344. 
[20] Pourahmadi, M. (1984). Taylor expansion of $\exp \left(\sum_{k=0}^{\infty} a_{k} z^{k}\right)$ and some applications. Amer. Math. Monthly 91, 303-307.

[21] R Development Core Team (2014). R: A Language and Environment for Statistical Computing.

[22] Soares, L. and Souza, L. (2006). Forecasting electricity demand using generalized long memory. Internat. J. Forecasting 22, 17-28.

[23] Talamantes, J., Behseta, S. and Zender, C. (2007). Statistical modeling of valley fever data in Kern County, California. Internat. J. Biometeorology 51, 307-313.

[24] Wikle, C. K., Sherman, P.J., and Chen, T-C. (1995). Identifying periodic components in atmospheric data using a family of minimum variance spectral estimators. Journal of Climate 8, 2352-2363.

[25] Woodward, W., Cheng, Q. and Gray, H. (1998). A k-factor GARMA long memory model. J. Time Ser. Anal. 19, 485-504. 